\title{
Supersolidity in quantum films adsorbed on graphene and graphite
}

\author{
M. C. Gordillo, ${ }^{1}$ C. Cazorla, ${ }^{2}$ and J. Boronat ${ }^{3}$ \\ ${ }^{1}$ Departamento de Sistemas Físicos, Químicos y Naturales, Facultad de Ciencias Experimentales, Universidad Pablo de Olavide, \\ Carretera de Utrera, km 1., E-41013 Sevilla, Spain \\ ${ }^{2}$ Institut de Ciència de Materials de Barcelona (ICMAB-CSIC), Campus UAB, E-08193 Bellaterra, Spain \\ ${ }^{3}$ Departament de Física i Enginyeria Nuclear, Universitat Politècnica de Catalunya, Campus Nord B4-B5, E-08034 Barcelona, Spain
}

(Received 22 February 2011; published 17 March 2011)

\begin{abstract}
Using quantum Monte Carlo we have studied the superfluid density of the first layer of ${ }^{4} \mathrm{He}$ and $\mathrm{H}_{2}$ adsorbed on graphene and graphite. Our main focus has been on the equilibrium ground state of the system, which corresponds to a registered $\sqrt{3} \times \sqrt{3}$ phase. The perfect solid phase of $\mathrm{H}_{2}$ shows no superfluid signal, whereas ${ }^{4} \mathrm{He}$ has a finite but small superfluid fraction $(0.67 \%)$. The introduction of vacancies in the crystal makes the superfluidity increase, showing values as large as $14 \%$ in ${ }^{4} \mathrm{He}$ without destroying the spatial solid order.
\end{abstract}

PACS number(s): 68.90.+g, 67.25.dp, 05.30.Jp, 67.80.bd

A supersolid state of matter is a fascinating possibility that has long attracted interest from both theoretical and experimental viewpoints. ${ }^{1,2}$ The simultaneous existence of spatial lattice order and off-diagonal long-range order defining a supersolid is rather counterintuitive and only a theoretical entelechy up to recent times. The old theoretical ideas put forward by the pioneering works of Andreev and Lifshitz ${ }^{3}$ and Chester $^{4}$ and Reatto ${ }^{5}$ have revived dramatically since the experimental findings of $\mathrm{Kim}$ and $\mathrm{Chan}^{6}$ in 2004 on the evidence of nonconventional moment of inertia in solid ${ }^{4} \mathrm{He}$ below some characteristic temperature around $100 \mathrm{mK}$. Whether these and some other similar experiments carried out by other teams are an unambiguous proof of the existence of supersolidity or not is still a matter of debate.

At present, much less is known on possible supersolid scenarios in two-dimensional (2D) ${ }^{7}$ or quasi-two-dimensional (Q2D) solid ${ }^{4} \mathrm{He}$. Helium atoms, when adsorbed on graphite or graphene, arrange sequentially in stacking layers that can be considered as nearly 2D systems. ${ }^{8}$ Interestingly, recent experiments carried out by Nyéki et al. ${ }^{9}$ in the second layer of ${ }^{4} \mathrm{He}$ adsorbed on graphite point to the existence of a $(\sqrt{7} \times \sqrt{7})$ commensurate solid phase that exhibits superfluid fractions as large as $20 \%$. However, recent path integral Monte Carlo (PIMC) work ${ }^{10}$ does not seem to support the existence of this phase, which was first predicted in simulations where the first layer of ${ }^{4} \mathrm{He}$ atoms was considered as inert. ${ }^{11}$ Nevertheless, the possibility of having a supersolid in a $2 \mathrm{D}$ environment, supported by Nyéki et al.'s observations, ${ }^{9}$ opens new and exciting avenues for the analysis of this new state of matter.

Recently, we have calculated the zero-temperature phase diagram of the first layer of ${ }^{4} \mathrm{He}$ (Ref. 12) and $\mathrm{H}_{2}$ (Ref. 13) adsorbed on graphene and graphite using quantum Monte Carlo (QMC) methods. Our results predict that the equilibrium ground state of both systems is a $\sqrt{3} \times \sqrt{3}$ commensurate phase, a conclusion which, in the case of graphite, is in agreement with other low-temperature simulations and experimental data. ${ }^{14-16}$ The aim of our previous studies was basically the determination of the energies of the different possible phases to draw the phase diagram and not the study of off-diagonal long-range order and/or superfluidity. To this end, we used a nonsymmetric wave function for describing the solid phases, an approach which obviously hinders any insight on the properties directly related to their Bose-Einstein statistics but which guarantees accurate evaluation of the energies due to the low-rate interparticle exchange. In this work, we are mainly concerned with the possibility of supersolidity in the first layer of ${ }^{4} \mathrm{He}$ and $\mathrm{H}_{2}$ adsorbed on graphene and graphite so that our methodology has been changed accordingly. Besides the characterization of the equilibrium ground-state phases, we have also analyzed the influence of vacancies in the superfluid response and energy of these films since point defects are indeed observed during quantum layer nucleation on carbon surfaces. It is worth mentioning that previous low-temperature attempts using PIMC have not found any signal of superfluidity in these layers ${ }^{10,11}$ although possible supersolidity induced by defects was not analyzed.

Since we are interested in possible ground-state supersolid phases of the first ${ }^{4} \mathrm{He}$ and $\mathrm{H}_{2}$ layers adsorbed on graphene and graphite, we use the diffusion Monte Carlo (DMC) method that, working at zero temperature, solves stochastically the Schrödinger equation of the $N$-particle system in an essentially exact way (within some statistical uncertainties). ${ }^{17}$ Zero-temperature approaches are especially adequate for these systems since the onset temperatures for supersolidity are, at least in bulk solids, very small $(50-100 \mathrm{mK})$, thus making finite-temperature approaches like PIMC of difficult use. The Hamiltonian of the system includes both interparticle $[V(r)]$ and particle-substrate $[U(r)]$ interactions,

$$
H=-\frac{\hbar^{2}}{2 m} \sum_{i=1}^{N} \nabla_{i}^{2}+\sum_{i<j}^{N} V\left(r_{i j}\right)+\sum_{i, J}^{N, N_{s}} U\left(r_{i J}\right),
$$

with capital indexes running on carbon substrate atoms and normal indexes on $\mathrm{He}$ atoms or $\mathrm{H}_{2}$ molecules. The ${ }^{4} \mathrm{He}-{ }^{4} \mathrm{He}$ and $\mathrm{H}_{2}-\mathrm{H}_{2}$ interactions are modeled by the accurate Aziz II (Ref. 18) and Silvera-Goldman ${ }^{19}$ potentials, respectively. The adsorbate surface potential is obtained by summing all the pair atom(molecule)-adsorbate interactions modeled by LennardJones potentials. By summing up all the latter pair interactions, we introduce in the description of the system the necessary corrugation to observe that a commensurate solid phase is effectively preferred for being the ground state. ${ }^{12,13}$

As it is usual in DMC, we introduce a trial wave function for importance sampling which improves the variance of the statistical estimation. Our variational model contains basic information: It is zero at shorter distances than the (hard-)core 
of the potential and becomes constant at large distances (Jastrow wave function); it is symmetric under the exchange of particles (Bose-Einstein statistics) and localizes particles in preferred points (sites) for solid phases. Explicitly, ${ }^{20}$

$$
\Psi(\boldsymbol{R})=\prod_{i<j}^{N} f\left(r_{i j}\right) \prod_{I=1}^{N_{\mathrm{cr}}}\left[\sum_{i=1}^{N} g\left(r_{I i}\right)\right] .
$$

In Eq. (2), $\boldsymbol{R}=\left\{\boldsymbol{r}_{1}, \ldots, \boldsymbol{r}_{N}\right\}, f(r)$ is a two-body Jastrow correlation factor, $g\left(r_{I i}\right)=\exp \left[-\alpha\left(\boldsymbol{r}_{i}-\boldsymbol{r}_{I}\right)^{2}\right]$, and $N_{\mathrm{cr}}$ is the number of lattice sites of the selected crystal structure. Model wave function (2) fulfills simultaneously spatial solid order and exchange-particle symmetry avoiding the numerically unworkable use of permanents on top of the nonsymmetric Nosanow-Jastrow wave function. The value of the variational parameters in $\Psi$ are the same as the ones reported in Refs. 12 and 13.

We have focused our attention in the $\sqrt{3} \times \sqrt{3}$ commensurate phase of ${ }^{4} \mathrm{He}$ and $\mathrm{H}_{2}$ since this is the equilibrium groundstate structure and best candidate for exhibiting supersolid behavior due to its quite low density. The simulation cell is a rectangle of fixed dimensions $44.27 \times 42.60 \AA^{2}$, where the number of sites of the registered phase is $N_{\text {cr }}=120$ and the number of particles is $115 \leqslant N \leqslant 120$; that is, we consider up to a maximum of five vacancies. The dependence of the energy per particle with the number of vacancies, reported as a function of the particle density, is shown in Fig. 1 for ${ }^{4} \mathrm{He}$ and $\mathrm{H}_{2}$ on top of graphene and graphite. For comparison
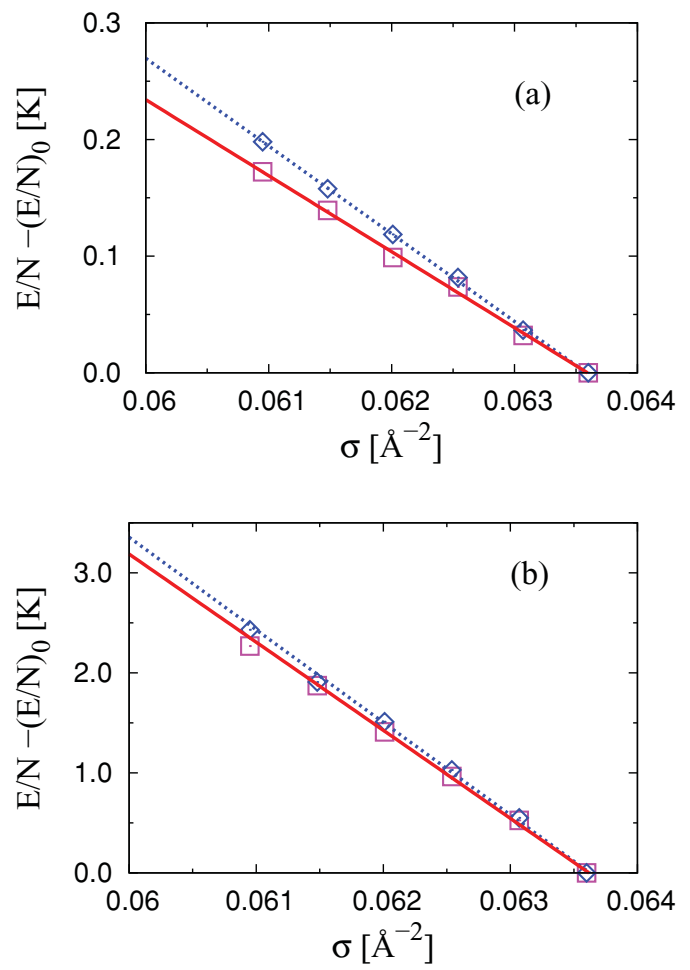

FIG. 1. (Color online) (a) Energy per ${ }^{4} \mathrm{He}$ atom with different number of vacancies, plotted as a function of the particle density. (b) Same as in (a) but for $\mathrm{H}_{2}$. In both cases the energy per particle of the commensurate phase with no vacancies $(E / N)_{0}$ is subtracted. Solid line and squares (dotted line and diamonds) stand for energies when the adsorbate is graphene (graphite). purposes, those curves have been displaced downward an energy shift $(E / N)_{0}$ which corresponds to the energy of the perfect structures reported in Refs. 12 and 13. For the present analysis it is enough to mention that the binding energy of $\mathrm{H}_{2}$ is approximately 2.5 times larger than that of ${ }^{4} \mathrm{He}$ and that in both systems the interactions with graphite are about $10 \%$ more attractive than with graphene.

As shown in Fig 1, the binding energies per particle increase systematically with the number of vacancies so the equilibrium ground state always corresponds to the perfect commensurate lattice. The variation of the activation vacancy energy is linear in both cases and slightly larger in graphite where the adsorption energies are invariably $\sim 10 \%$ larger than in graphene. Comparing the results obtained for ${ }^{4} \mathrm{He}$ and $\mathrm{H}_{2}$, one can see that the energy difference $E / N-(E / N)_{0}$ is roughly 10 times larger for hydrogen; however, as the absolute values for both solids are so different, it is better to make a comparison in relative terms: The energy cost of creating five vacancies in ${ }^{4} \mathrm{He}$ is $0.15 \%$ of the ground-state energy while in $\mathrm{H}_{2}$ is $0.43 \%$. When the number of vacancies increases, and therefore the particle density decreases, the equation of state of the solid with vacancies approaches the equation of state of the metastable liquid phase. This analysis is especially interesting when graphene is the substrate since the difference in binding energy at the equilibrium point of the liquid and the one at the commensurate solid phase is very tiny, nearly 4 times smaller than in graphite. For both ${ }^{4} \mathrm{He}$ and $\mathrm{H}_{2}$, all the energies shown in Fig. 1 are below the ones calculated for the corresponding liquid phases at the same densities. If the linear behavior observed in the figure is extrapolated to smaller densities, one can see that in ${ }^{4} \mathrm{He}$ the intersection with the liquid equation of state is produced at density $\sigma_{\mathrm{c}}=0.058 \AA^{-2}$, which would correspond to 10 vacancies in the simulation box. In this case, $\sigma_{\mathrm{c}}$ is larger than the equilibrium density of the liquid $\sigma_{0}=0.044 \AA^{-2}$; thus, the crossing point will appear at finite pressure. In contrast, the intersection with the liquid equation of state in hydrogen appears at density $\sigma_{\mathrm{c}}=0.052 \AA^{-2}$, which is smaller than the equilibrium point $\sigma_{0}=0.059 \AA^{-2}$ and thus corresponds to the metastable negative-pressure regime.

A discussion on possible supersolid phases of helium and hydrogen on top of graphene or graphite requires the estimation of the superfluid density fraction of the different solid phases that appear in their respective phase diagrams. In QMC, the superfluid fraction can be computed by sampling the winding number ${ }^{21}$

$$
\boldsymbol{W}=\sum_{i=1}^{N} \int_{0}^{\beta} d \tau\left(\frac{d \boldsymbol{r}_{i}(\tau)}{d \tau}\right),
$$

with $\tau$ the imaginary time and $\beta=T^{-1}$. In the limit of zero temperature $\beta \rightarrow \infty$, the winding number (3) turns to the diffusion coefficient of the center of mass of the $N$ particles $\left(\boldsymbol{R}_{C M}\right)$ in the simulation box with periodic boundary conditions, ${ }^{22}$

$$
\frac{\rho_{\mathrm{s}}}{\rho}=\lim _{\tau \rightarrow \infty} \alpha\left(\frac{D_{\mathrm{s}}(\tau)}{\tau}\right),
$$

where $\alpha=N / 2 d D_{0}$, with $d$ the number of dimensions $(d=2$ in the present case), $D_{0}=\hbar^{2} / 2 m$, and $D_{\mathrm{s}}(\tau)=\left\langle\left[\boldsymbol{R}_{C M}(\tau)-\right.\right.$ $\left.\left.\boldsymbol{R}_{C M}(0)\right]^{2}\right\rangle$. The diffusion coefficient $D_{\mathrm{s}}(\tau)$ can be calculated 


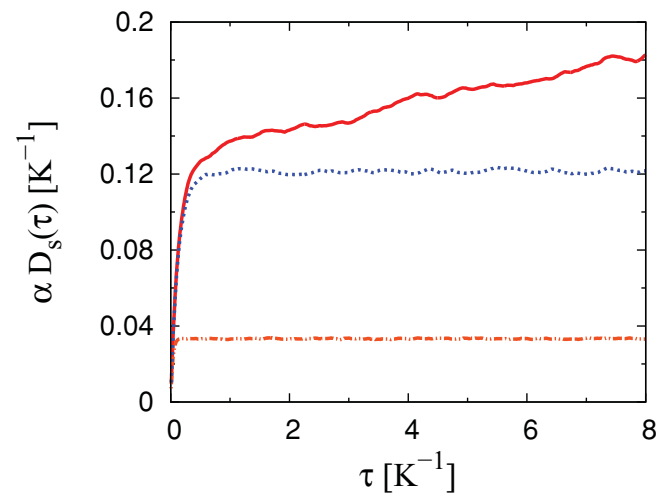

FIG. 2. (Color online) DMC estimation of the superfluid density. Solid, dotted, and dashed lines stand for commensurate ${ }^{4} \mathrm{He}$, incommensurate ${ }^{4} \mathrm{He}$, and commensurate $\mathrm{H}_{2}$ phases on top of graphene, respectively.

using the DMC method, in which the imaginary time evolves in a continuous way, and it can be proved that in the asymptotic regime this estimator is unbiased with respect to the particular choice of the trial wave function used for importance sampling (within a specified physical phase). According to the typical statistical noise in DMC simulations, the resolution of this estimator is much lower $\left(\sim 1 \times 10^{-5}\right)$ than in PIMC, where superfluid signals below $\sim 1 \times 10^{-2}$ are extremely difficult to be measured.

In Fig. 2, results for $\alpha D_{\mathrm{s}}(\tau)$ as a function of the imaginary time $\tau$ are shown. As obvious from its definition (4), a
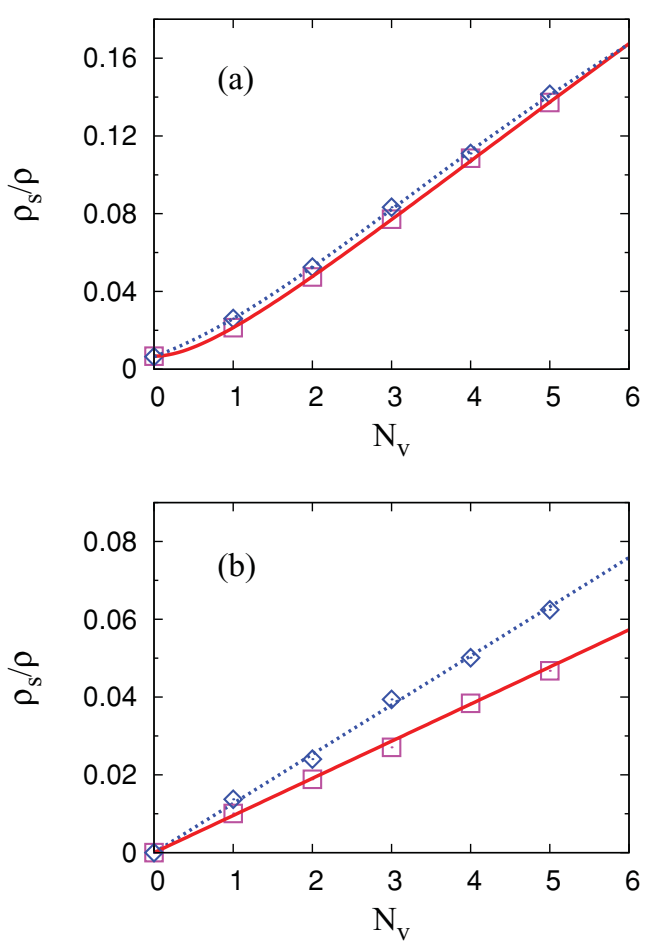

FIG. 3. (Color online) (a) Superfluid fraction of the ${ }^{4} \mathrm{He}$ commensurate solid phase with different number of vacancies $N_{v}$. (b) Same as in (a) but for $\mathrm{H}_{2}$. Solid line and squares (dotted line and diamonds) stand for superfluid fractions when the adsorbate is graphene (graphite). finite superfluid fraction appears as a finite slope in the long-time behavior of the diffusion coefficient $D_{\mathrm{s}}(\tau)$, their particular values being not relevant and fairly dependent of the kind of system under study. Our results for the perfect (no vacancies) solid phases plotted in Fig. 2 show different behaviors depending on the system and solid phases considered. Diffusion coefficient results for long time $\tau$ obtained for the $\sqrt{3} \times \sqrt{3}$ commensurate phase of ${ }^{4} \mathrm{He}$ on top of graphene (and also in graphite) show a small but clear slope $\left[\rho_{s} / \rho=0.0067(1)\right]$ that contrasts with the null $\tau$ variation observed in a incommensurate phase of density $0.0999 \AA^{-2}$. Interestingly, simulations performed in the commensurate phase of $\mathrm{H}_{2}$ indicate zero superfluid fraction, thus areal density must be ruled out as the only cause behind supersolidity in quantum films. Moreover, as it was shown in the DMC calculation of 2D and quasi-2D ${ }^{4} \mathrm{He}$ in Ref. 23, the superfluid fraction of a purely $2 \mathrm{D}$ crystal is zero even at densities below the $\sqrt{3} \times \sqrt{3}$ phase, and finite superfluidity emerges only with the opening of a transverse direction that particles can explore. According to these previous results, the zero signal observed in $\mathrm{H}_{2}$ can be explained in terms of transverse motion frustration resulting from intense molecular binding to carbon surfaces.

One of the aims of this work has been the study of the influence of the number of vacancies in the superfluid fraction of quantum solid layers. To this end, we have calculated $\rho_{s} / \rho$ for the commensurate phase of ${ }^{4} \mathrm{He}$ and $\mathrm{H}_{2}$ on top of graphene and graphite with up to five $\left(N_{v}\right)$ vacancies within a total of 120 possible sites of our simulation cell. The results, shown in Fig. 3, show a linear increase of the superfluid fraction with $N_{v}$
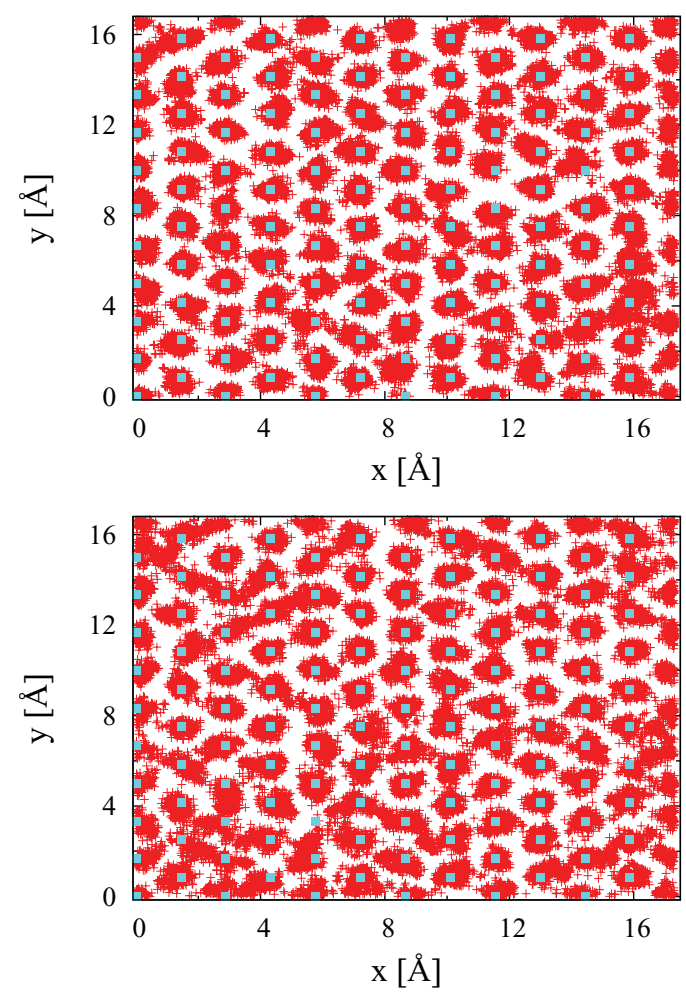

FIG. 4. (Color online) (Top) Snapshot of the probability density (crosses) of commensurate solid ${ }^{4} \mathrm{He}$ on top of graphene. (Bottom) Same as in the top panel but with five fewer particles in the simulation box. In both figures, squares are the sites of the perfect crystal. 
for both helium and hydrogen. The increase of superfluidity with $N_{v}$ is significantly larger for ${ }^{4} \mathrm{He}$; for instance, in the $N_{v}=5$ case $\rho_{s} / \rho$ amounts to 0.14 and 0.047 for ${ }^{4} \mathrm{He}$ and $\mathrm{H}_{2}$, respectively. The effect of the substrate on the superfluid fraction is smaller in helium than in hydrogen but in both cases $\rho_{s} / \rho$ is always larger in graphite than in graphene. The difference is very small in ${ }^{4} \mathrm{He}$ but significant in $\mathrm{H}_{2}$. A possible explanation of the increase of superfluidity in graphite relies on the slightly reduced motion in the transverse direction due to stronger attraction with respect to graphene. This increase in confinement makes more effective the motion of the center of mass of the system in the $x-y$ plane of the simulation box, where large particle-permutational rings can be created, and consequently $\rho_{s} / \rho$ increases (4).

We have verified that in both hydrogen and helium the periodic spatial order characteristic of the solid phase is conserved up to five vacancies in 120 possible sites. This is shown in Fig. 4, where characteristic snapshots of DMC simulations are shown for the perfect and defective $N_{v}=5$ film phases $\left({ }^{4} \mathrm{He}\right)$. These snapshots correspond to the probability density that in DMC is represented by a collection of walkers, each one of $3 N$ coordinates, that evolves in imaginary time according to the Schrödinger equation. As one can see, even in the case of the perfect crystal there is a finite probability of visiting the intersite space, which can be interpreted as the finite exchange probability leading to nonzero superfluid signal (a quantitative estimation of probability exchange would require of specific methods beyond the scope of the present work $\left.{ }^{24}\right)$. When vacancies are present in the system the paths connecting different sites become more populated, that is, the superfluidity increases, but solid order remains preserved. It is worth noticing that in the snapshot of the layer with vacancies one cannot allocate the vacancies implying that they have become indistinguishable. Same snapshots for $\mathrm{H}_{2}$ show that in the perfect crystal the intersite occupation is zero (zero superfluidity) and that the vacancies are identified more easily than in helium.

Summarizing, using the DMC method we have studied the supersolidity of the first layer of ${ }^{4} \mathrm{He}$ and $\mathrm{H}_{2}$ adsorbed on graphene and graphite in the limit of zero temperature. The ${ }^{4} \mathrm{He} \sqrt{3} \times \sqrt{3}$ commensurate phase shows a small but finite superfluid signal $(0.67 \%)$, whereas the $\mathrm{H}_{2}$ one does not within our resolution limit $\left(1 \times 10^{-5}\right)$. When vacancies are present in the system, the superfluid fraction increases with the concentration of defects; this effect is larger in helium where we have obtained values as large as $\rho_{s} / \rho=14 \%$. As the presence of point defects in quantum layers is plausible due to imperfections in the adsorbent surfaces, further experiments on quasi-2D systems can lead to the emergence of new and tunable supersolid scenarios..$^{9,25}$

Authors would like to thank Jan Nyéki for helpful discussions. We acknowledge partial financial support from the Junta de Andalucía group PAI-205, DGI (Spain) Grant No. FIS2008-04403, and Generalitat de Catalunya Grant No. 2009SGR-1003.
${ }^{1}$ S. Balibar and F. Caupin, J. Phys. Condens. Matter 20, 173201 (2008).

${ }^{2}$ N. Prokof'ev, Adv. Phys. 56, 381 (2007).

${ }^{3}$ A. F. Andreev and I. M. Lifshitz, Sov. Phys. JETP 29, 1107 (1969).

${ }^{4}$ G. V. Chester, Phys. Rev. A 2, 256 (1970).

${ }^{5}$ L. Reatto, Phys. Rev. 183, 334 (1969).

${ }^{6}$ E. Kim and M. H. W. Chan, Science 305, 1941 (2004); Nature (London) 427, 225 (2004).

${ }^{7}$ M. Rossi, E. Vitali, D. E. Galli, and L. Reatto, J. Phys. Condens. Matter 22, 145401 (2010).

${ }^{8}$ L. W. Bruch, M. W. Cole, and E. Zaremba, Physical Adsorption: Forces and Phenomena (Oxford Science Publishers, Oxford, 1997). ${ }^{9}$ Communication presented by J. Nyéki et al., to the workshop Supersolids 2009 held in Banff (Canada).

${ }^{10}$ P. Corboz, M. Boninsegni, L. Pollet, and M. Troyer, Phys. Rev. B 78, 245414 (2008).

${ }^{11}$ M. Pierce and E. Manousakis, Phys. Rev. Lett. 81, 156 (1998).

${ }^{12}$ M. C. Gordillo and J. Boronat, Phys. Rev. Lett. 102, 085303 (2009).
${ }^{13}$ M. C. Gordillo and J. Boronat, Phys. Rev. B 81, 155435 (2010).

${ }^{14}$ M. Bretz and J. G. Dash, Phys. Rev. Lett. 27, 647 (1971).

${ }^{15}$ M. Bretz, J. G. Dash, D. C. Hickernell, E. O. McLean, and O. E. Vilches, Phys. Rev. A 8, 1589 (1973).

${ }^{16}$ J. G. Dash, Phys. Rev. B 15, 3136 (1977).

${ }^{17}$ J. Boronat and J. Casulleras, Phys. Rev. B 49, 8920 (1994).

${ }^{18}$ R. A. Aziz, F. R. W. McCourt, and C. C. K. Wong, Mol. Phys. 61, 1487 (1987).

${ }^{19}$ I. F. Silvera and V. V. Goldman, J. Chem. Phys. 69, 4209 (1978).

${ }^{20}$ C. Cazorla, G. E. Astrakharchik, J. Casulleras, and J. Boronat, New J. Phys. 11, 013047 (2009).

${ }^{21}$ D. M. Ceperley, Rev. Mod. Phys. 67, 279 (1995).

${ }^{22}$ S. Zhang, N. Kawashima, J. Carlson, and J. E. Gubernatis, Phys. Rev. Lett. 74, 1500 (1995).

${ }^{23}$ C. Cazorla, G. E. Astrakharchik, J. Casulleras, and J. Boronat, J. Phys. Condens. Matter 22, 165402 (2010).

${ }^{24}$ D. M. Ceperley, Rev. Mod. Phys. 67, 279 (1995).

${ }^{25}$ Y. Shibayama, H. Fukuyama, and K. Shirahama, J. Phys. Conf. Ser. 150, 032096 (2009). 